\title{
Age-associated decrease in global DNA methylation in patients with major depression
}

This article was published in the following Dove Press journal:

Neuropsychiatric Disease and Treatment

10 November 2014

Number of times this article has been viewed

\section{Ping-Tao Tseng ${ }^{1,2, *}$ \\ Pao-Yen Lin ${ }^{1,3, *}$ \\ Yu Lee' \\ Chi-Fa Hung' \\ For-Wey Lung 4,5 \\ Cheng-Sheng Chen ${ }^{6,7}$ \\ Mian-Yoon Chong'}

'Department of Psychiatry, Kaohsiung Chang Gung Memorial Hospital and Chang Gung University College of Medicine, Kaohsiung, Taiwan; ${ }^{2}$ Department of Psychiatry, TsyrHuey Mental Hospital, Kaohsiung Jen-Ai's Home, Taiwan; ${ }^{3}$ Center for Translational Research in Biomedical Sciences, Kaohsiung Chang Gung Memorial Hospital, Kaohsiung, Taiwan; ${ }^{4}$ Taipei City Psychiatric Center, Taipei City Hospital, Taipei, Taiwan; ${ }^{5}$ Graduate Institute of Medical Science, National Defense Medical Center, Taipei, Taiwan; ${ }^{6}$ Department of Psychiatry, Kaohsiung Medical University Hospital, Kaohsiung, Taiwan; ' ${ }^{7}$ Department of Psychiatry, College of Medicine, Kaohsiung Medical University, Kaohsiung, Taiwan

*These authors contributed equally to this work
Correspondence: Pao-Yen Lin Department of Psychiatry, Kaohsiung Chang Gung Memorial Hospital, 123, Dapi Road, Niaosong District, Kaohsiung City 833, Taiwan

Tel +88677317123 ext 875 I

Fax +88677326817

Email py1029@adm.cgmh.org.tw
Background: Evidence has supported a role of DNA methylation in the pathophysiology of mood disorders. The purpose of the current study is to examine 5-methylcytosine $(5-\mathrm{mc})$ and 5-hydroxymethylcytosine (5-hmc) levels in patients with major depressive disorder (MDD) at different disease states.

Methods: Forty-nine patients with MDD and 25 healthy control subjects were included. The severity in the disease was assessed by using the 17-item Hamilton Rating Scale of Depression (HAM-D) (HAM-D $\geq 19$ for severe MDD and HAM-D $\leq 7$ for remitted MDD). The 5-mc and 5-hmc levels in leukocyte DNA were measured using an enzyme-linked immunosorbent assay-based method.

Results: We found a significant decrease in 5-hmc and trends of decreasing 5-mc levels in patients with severe MDD compared to healthy controls ( $P=0.059$ for 5 -mc and $P=0.013$ for 5 -hmc). The decrease in the level exists only in the older age group $(P=0.035$ for 5 -mc and $P=0.002$ for 5 -hmc) but not in the younger age group ( $P=0.077$ for 5 -mc and $P=0.620$ for 5 -hmc). In addition, the 5-mc level was found to be inversely correlated with disease severity $(P=0.011)$.

Conclusion: Our results support a decrease in global DNA methylation associated with age in patients with severe depression. Further studies are needed to clarify the role of the methylation level as a disease marker of depression and whether antidepressant treatment changes the methylation profiles.

Keywords: 5-methylcytosine, 5-hydroxymethylcytosine, antidepressant, mood disorder, gene modification, epigenetic

\section{Introduction}

Although evidence has shown a heightened risk of illness in the siblings of patients with depression, the heritability of depression was estimated to be only one-third. ${ }^{1}$ Cross-generational transmission of gene sequence information does not seem to explain all the etiology or susceptibility of depression. Some environmental factors, including early trauma, daily life stressors, and acquired substance abuse, may play some role in the development of depression. Epigenetic regulation, concerning mechanisms that regulate gene expression beyond the gene sequence, has been proposed to explain the gene-environment interaction in the susceptibility to depression. ${ }^{2}$

A significant effect in maternal behavior has been found on the methylation of a glucocorticoid receptor gene promoter in the offspring. The change in the methylation level can result in altered hypothalamic-pituitary-adrenal response to stress, and these changes can be reversed by giving histone deacetylase inhibitors. ${ }^{3}$ This provided strong evidence showing that an epigenetic state of a gene can be established through behavioral programming. In parallel, it was also found that childhood maltreatment 
resulted in hypermethylation of a glucocorticoid receptor gene (NR3C1) promoter, across patients with various psychiatric diagnoses. ${ }^{4}$ In addition, the severity, the number, and the type of maltreatments positively correlated with the level of methylation. ${ }^{4}$ Recently, Jiao et al found that the modification of methylation levels of $\alpha$-calcitonin-gene-related peptide promoter can induce or reduce the depression-like behaviors. ${ }^{5}$ In spite of the evidence of the relationship between methylation profiles and depression in animal models, there is still limited evidence showing the change of methylation levels in clinical subjects with depression. ${ }^{6-9}$ The contribution of epigenetic mechanisms, including gene methylation and histone modifications, on the pathogenesis of depression has been extensively reviewed elsewhere. ${ }^{10,11}$

Methylation of the 5-position of cytosine base into 5-methylcytosine (5-mc) in DNA, catalyzed by DNA methyltransferase (DNMT), is one of the major epigenetic processes in eukaryotes. ${ }^{12}$ The 5 -mc was believed to exert a silencing effect on DNA transcription through accumulation of mutation. ${ }^{12}$ However, there is still some inconsistency about the physiologic role of 5-mc in gene transcription. For example, it has been shown that the presence of 5-mc at nonpromoter $\mathrm{CpG}$ islands is important for the maintenance of the active chromatic state critical for neurogenesis. ${ }^{13}$ On the other hand, 5-hydroxymethylcytosine $(5-\mathrm{hmc})$ is a product of conversion from 5-mc catalyzed by the ten-eleven translocation 1 (TET1) 5-mc oxidase. ${ }^{14}$ The 5 -hmc is believed to have lower affinity to the methyl-CpG binding domain of methyl-CpG binding protein 2 (MeCP2), ${ }^{15}$ which can recruit cytosine methyltransferases, histone deacetylases, and other chromatin remodeling proteins. Therefore, 5-hmc can initiate the epigenetic regulation, such as histone methylation and deacetylation. ${ }^{16-18}$ Also, conversion from 5-mc to 5-hmc prevents the DNMT1 methylation process of targeted cytosine; ${ }^{19}$ hence, facilitating passive DNA demethylation by interfering with the maintenance role of DNMT1. ${ }^{14}$ In summary, 5-hmc may hinder 5-mc-targeted repression of gene transcription, and may play a role in potentiating gene regulation. ${ }^{20}$ When looking at neuronal function, it was also recently found out that neuronal TET1 enzyme regulates normal DNA methylation levels, expression of activityregulated genes, hippocampal long-term depression, and memory extinction. ${ }^{21}$

Many clinical studies have found decreased 5-hmc levels in many types of cancer cells. ${ }^{22-24}$ For example, it has been shown that the loss of 5-hmc levels is associated with increased melanoma virulence, whereas rebuilding the 5-hmc landscape in melanoma cells by reintroducing active TET2 suppresses melanoma growth and increases tumor-free survival in animal models. ${ }^{25}$ However, there are only a few reports mentioning the 5 -hmc or 5-mc expression in the nervous system. Recently, it was found that aging decreased mouse mitochondrial DNA 5-hmc levels in the frontal cortex, but not 5-mc levels. ${ }^{26}$ In another study, global hippocampal 5 -hmc content was seen to increase during aging in the absence of 5-mc decrease. ${ }^{27}$ So far, there are only a few published studies examining 5-mc levels in patients with depression. Nagy et al recently found differentially methylated regions of DNA in astrocytes of prefrontal cortex between depressive cases and control subjects, whereas the majority of those regions displayed reduced levels in the methylation levels in patients. ${ }^{28}$ Davies et al reported an investigation on the methylation levels in the whole blood of monozygotic twins, which found significant hypermethylation within the coding region of $Z B T B 20$ in patients with a major depressive disorder (MDD). ${ }^{29}$ However, no study examining the 5-hmc level has been published so far.

In this study, we examine the levels of global gene methylation in peripheral leukocytes in patients with clinical depression. The goal of the study is to compare the levels of 5-mc and 5-hmc in patients with MDDs with different disease states with those of healthy control subjects.

\section{Material and methods Subjects}

Subjects were recruited following the protocol described in Figure 1. Inpatients or outpatients meeting the Diagnostic and Statistical Manual of Mental Disorders 4th edition text revision (DSM-IV-TR) criteria of MDD were consecutively recruited. The diagnosis was conducted by a well-trained psychiatrist (Y Lee or P-Y Lin) using the Structured Clinical Interview for DSM-IV (SCID-IV). The subject recruitment process was similar to that described in our previous study. ${ }^{30}$ The 83 recruited MDD patients were next screened by the following criteria: 1) age between 20 years and 70 years; 2) 17-item Hamilton Rating Scale for Depression $($ HAM-D) $\geq 19$ or $\leq 7$; 3) no major systemic disease, head trauma, cancer, or neurological disorder; and 4) no history of dementia, delirium, or substance or alcohol abuse. In the inclusion criteria of the MDD subjects, we defined those with HAM-D $\leq 7$ as remitted depression according to the criteria used in the reports by Dunner et $\mathrm{al}^{31}$ and Arnold et al. ${ }^{32} \mathrm{On}$ the other hand, because there are inconsistent criteria for active or severe depression in Asian subjects (eg, HAM-D $\geq 15$ in one report from Korea, ${ }^{33}$ and HAM-D $\geq 17$ in one report from China), ${ }^{34}$ we chose the criteria of HAM-D score as $\geq 19$ 
83 patients meeting DSM-IV-TR MDD diagnosis were recruited

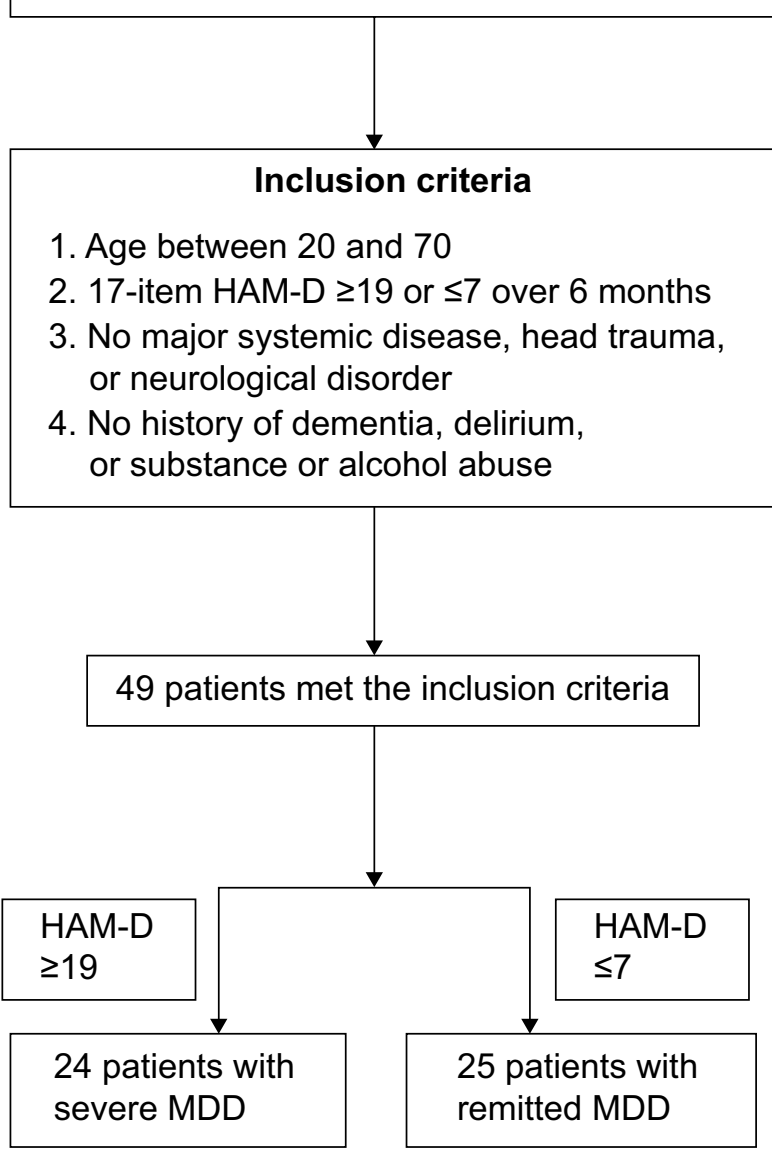

132 healthy control subjects recruited from a general health examination center

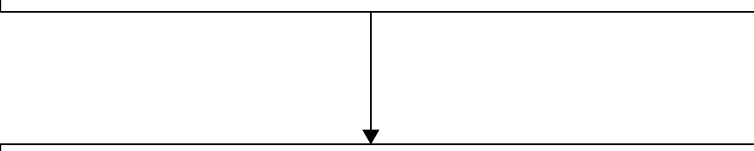

Inclusion criteria

1. Age between 20 and 70

2. $\mathrm{CHQ}<4$

3. No history of psychiatric disorders, suicide attempt, substance or alcohol abuse

4. No major systemic disease, head trauma, neurological disorder, dementia, or delirium

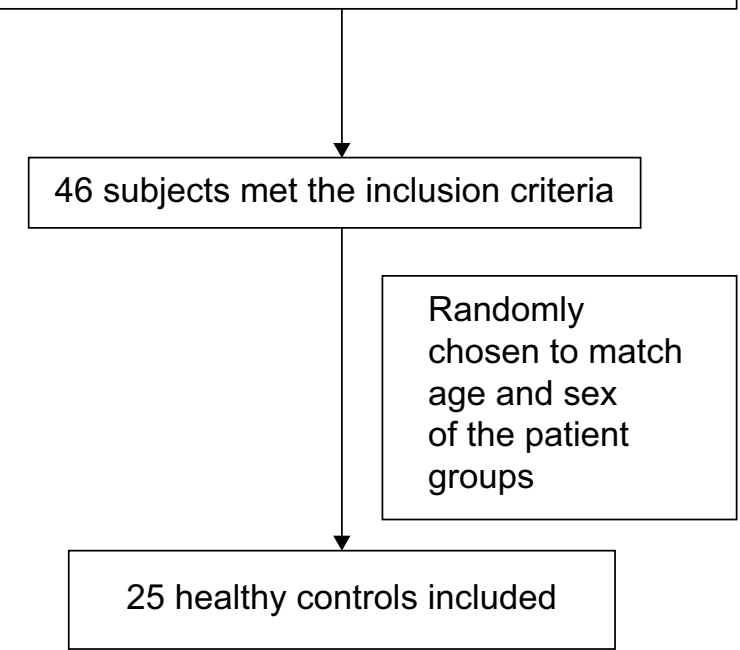

Figure I Subject recruitment procedure in current study.

Abbreviations: CHQ, Chinese Health Questionnaire; DSM-IV-TR, Diagnostic and Statistical Manual of Mental Disorders 4th edition text revision; HAM-D, Hamilton Rating Scale for Depression; MDD, major depressive disorder.

for the severe MDDs according to our previous report. ${ }^{30}$ Healthy control subjects were recruited from a general health examination center and screened using the following inclusion criteria: 1) age between 20 years and 70 years; 2 ) 12-item Chinese Health Questionnaire (CHQ) $<4$; and 3) no history of psychiatric disorders, suicide attempts, substance or alcohol abuse, major systemic disease (including metabolic diseases), head trauma, cancer, neurological disorder, dementia, or delirium. The CHQ was designed for identification of minor psychiatric disorders in both community and hospital subjects. ${ }^{35}$ Its sensitivity and specificity could reach as high as 0.78 and 0.77 , respectively, with optimal cutoff point for the best compromise between high sensitivity and a low false-positive rate as $3 / 4 .{ }^{36} \mathrm{We}$ excluded control subjects with CHQ score higher than 3. Next, all the healthy controls meeting the criteria were randomly chosen to match age and sex of the MDD patients group. All the subjects included in our study were of Han Chinese origin. This study was approved by the Institutional Review Board of Chang Gung Memorial Hospital, and informed consent was obtained from each participant. Finally, 74 subjects ( 25 healthy control subjects, 24 severe MDD patients, and 25 remitted MDD patients) were included in our study.

In our subclassification system of MDD patients, the drug-free patients were defined as those not receiving any psychotropic agents for at least 3 months. Treatment-resistant depression (TRD) was defined by the criteria as MDD with poor response to two adequate trials of different classes of antidepressants. ${ }^{37}$ In order to compare the effect on genome methylation, we transformed the antidepressant dosage into imipramine equivalents and antipsychotic dosage into chlorpromazine equivalents according to previous reports. ${ }^{38,39}$

It had been shown that 5-mc in leukocytes decreased with age. ${ }^{40,41}$ Furthermore, one previous study had shown 
that there was a sex-specific negative correlation between the level of 5-mc and age. ${ }^{42}$ To examine the age-associated difference in 5-mc and 5-hmc, we divided all the subjects according to their age, as in our previous study. ${ }^{30}$ Then we coded them as younger-age severe MDD patients subgroup (YSPS) and younger-age remitted MDD patients subgroup (YRPS) with equal to or below 35 years of age, middle-aged severe MDD patients subgroup (MSPS) and middle-aged remitted MDD patients subgroup (MRPS) with 36-50 years, and older severe MDD patients subgroup (OSPS) and older remitted MDD patients subgroup (ORPS) with over 50 years. Similarly, young healthy control subgroup (YHS), middleaged healthy control subgroup (MHS), and older healthy control subgroups (OHS) were subdivided in the same age ranges. In addition, moderating effect by sex of the patients on 5-mc and 5-hmc levels was also examined.

\section{Blood sample preparation and 5-mc and 5-hmc analysis}

Genomic DNA was extracted from peripheral leukocytes by using a commercial kit (QIAGEN, Valencia, CA, USA). MethylFlash $^{\mathrm{TM}}$ methylated DNA quantification kit and MethylFlash $^{\mathrm{TM}}$ hydroxymethylated DNA quantification kit (Epigentek, Farmingdale, NY, USA) were used to measure the percentage of 5-mc and 5-hmc, respectively. Both are enzyme-linked immunosorbent assay-based colorimetric assays. The procedures were performed according to the protocols provided by the manufacturer. All assays were conducted in duplicate. Average 5-mc and 5-hmc of the duplicates were used in subsequent analyses.

\section{Statistical analysis}

Continuous variables were presented as means \pm standard deviation (SD). All the statistical analyses were performed by using the Statistical Package for the Social Sciences (SPSS) version 11.0 for Windows. A $P$-value $<0.05$ was considered to be significant. The chi-squared analysis was performed on categorical data. We used a two-tailed $t$-test or analysis of variance (ANOVA) with Scheffe post hoc test to compare continuous variables. We also tested the correlation between 5-mc and 5-hmc levels, and these levels with some factors, such as age, illness duration, illness onset, education, body mass index (BMI), HAM-D scores, chlorpromazine equivalents, and imipramine equivalents, by the Pearson correlation test.

We compared 5-mc and 5-hmc levels between MDD patients and healthy subjects with the same age (eg, YSPS/ YRPS vs YHS) by using a two-tailed $t$-test. Also, ANOVA with Scheffe post hoc test was used to compare 5-mc and 5 -hmc levels in the three age groups in patients and healthy controls. We also performed two-tailed $t$-tests to compare 5-mc and 5-hmc levels between MDD patients with first episode and MDD patients with recurrent episodes, and also between TRD and non-TRD patients.

\section{Results}

\section{Demographic data and clinical variables}

A summary of the demographic data and clinical characteristics of our research subjects is shown in Table 1. There was no significant difference in sex distribution $(P=0.104)$, age $(P=0.614)$, or BMI $(P=0.579)$ among healthy controls,

Table I Demographic and clinical characteristics of subjects included in current study

\begin{tabular}{|c|c|c|c|c|}
\hline & $\begin{array}{l}\text { Healthy controls } \\
(n=25)\end{array}$ & $\begin{array}{l}\text { Severe MDD patients } \\
(n=24)\end{array}$ & $\begin{array}{l}\text { Remitted MDD patients } \\
(n=25)\end{array}$ & P-value \\
\hline Sex proportion (\% of females) & 44.0 & 70.8 & 64.0 & $0.104^{a}$ \\
\hline Age (years) & $48.3 \pm 11.1$ & $45.9 \pm 11.5$ & $49.2 \pm 13.2$ & $0.614^{b}$ \\
\hline Years of education (years) & $14.6 \pm 3.3$ & $10.2 \pm 4.1$ & $9.4 \pm 5.2$ & $<0.00 \mathrm{I}^{\mathrm{b}}$ \\
\hline BMI $\left(\mathrm{kg} / \mathrm{m}^{2}\right)$ & $23.8 \pm 3.7$ & $24.6 \pm 3.6$ & $23.5 \pm 4.2$ & $0.579^{b}$ \\
\hline Onset of illness (years) & NA & $35.8 \pm 12.2$ & $43.7 \pm 12.7$ & $0.03 I^{c}$ \\
\hline Duration of illness (months) & NA & $100.8 \pm 101.1$ & $66.2 \pm 53.1$ & $0.152^{c}$ \\
\hline HAM-D & NA & $25.5 \pm 4.4$ & $3.0 \pm 2.1$ & $<0.00 \mathrm{I}^{\mathrm{C}}$ \\
\hline Imipramine equivalence (mg) & NA & $120.1 \pm 43.9$ & $126.0 \pm 55.8$ & $0.736^{c}$ \\
\hline Chlorpromazine equivalence (mg) & NA & $247.6 \pm 191.3$ & $51.7 \pm 31.4$ & $0.035^{c}$ \\
\hline Previous suicide history (\%) & NA & 50.0 & 16.0 & $0.025^{\mathrm{a}}$ \\
\hline Family history of psychiatric disorder (\%) & NA & 45.8 & 32.0 & $0.484^{a}$ \\
\hline Family history of suicidal behavior (\%) & NA & 20.8 & 20.0 & $0.777^{a}$ \\
\hline 5-methylcytosine (\%) & $1.45 \pm 0.6 \mathrm{I}$ & $1.15 \pm 0.32$ & $1.35 \pm 0.31$ & $0.053^{b}$ \\
\hline 5-hydroxymethylcytosine (\%) & $2.05 \pm 1.02$ & $1.44 \pm 0.49$ & $1.45 \pm 0.45$ & $0.004^{b}$ \\
\hline
\end{tabular}

Notes: Data are shown as mean \pm standard deviation. ${ }^{a}$ Chi-square test. ${ }^{b}$ One-way ANOVA test. Independent-sample $t$-test.

Abbreviations: BMI, body mass index; HAM-D, Hamilton Depression Rating Scale; MDD, major depressive disorder; NA, not applicable. 
severe MDD patients, and remitted MDD patients. There was also no significant difference in duration of illness ( $P=0.152)$, imipramine equivalence $(P=0.736)$, family history with psychiatric disorders $(P=0.484)$, and family suicide history $(P=0.777)$ between remitted and severe MDD groups. However, the healthy controls had significantly more "years of education" than the two patient groups (compared with severe MDD, $P=0.003$; compared with remitted MDD, $P<0.001)$. The patients with severe MDD had significantly earlier illness onset $(P=0.031)$ and significantly more chlorpromazine equivalence $(P=0.035)$ than the patients with remitted MDD. There were nine drug-free patients in the severe MDD but only one in the remitted MDD group. In addition, there were seven first-episode MDD patients in the severe MDD and ten in the remitted MDD group. All six TRD patients were in the severe MDD group.

\section{5-mc and 5-hmc levels in healthy controls, severe MDD patients, and remitted MDD patients}

The 5-mc and 5-hmc levels in healthy controls, severe MDD patients, and remitted MDD patients are presented in Table 1, with a significant difference found for 5 -hmc $(F=6.092, d f=2$, $P=0.004)$ levels among three groups and a trend of difference found for 5-mc $(F=3.058, d f=2, P=0.053)$ levels among three groups (Table 1). In the post hoc test, the level of 5-mc in patients with severe MDD had trends of lower level than controls $(P=0.059)$, but there was no difference between control subjects and remitted MDD patients $(P=0.731)$ (Figure 2A). The level of 5-hmc in healthy controls was significantly higher than in both patients with severe $\operatorname{MDD}(P=0.013)$ and patients with remitted MDD $(P=0.014)$ (Figure 2B). However, we did not find any significant difference between the two patient groups in both 5-mc and 5-hmc $(P=0.266$ and $P=0.998$, respectively).

\section{Effects of age on the levels of 5-mc and 5-hmc levels}

The number of subjects in each group is as follows: four in the YHS, five in the YSPS, and five in the YRPS; ten in the MHS, ten in the MSPS, nine in the MRPS; eleven in the OHS, nine in the OSPS, and eleven in the ORPS. We would like to clarify the effects of disease status on 5-mc and 5-hmc levels in different age groups. We found significantly higher 5 -mc levels in the OHS than in the OSPS $(1.68 \% \pm 0.47 \%$ in OHS, $1.19 \% \pm 0.34 \%$ in OSPS, $P=0.035)$ but not with the ORPS ( $1.29 \% \pm 0.35 \%$ in ORPS, $P=0.090)$. Between OSPS and ORPS, there was also no significant difference $(P=0.846)$ (Figure 3A). For 5-hmc levels, similarly, we found significant higher levels in OHS than in OSPS and ORPS (2.52\% $\pm 0.76 \%$ in OHS, $1.45 \% \pm 0.53 \%$ in OSPS, $1.37 \% \pm 0.49 \%$ in ORPS; $P=0.002$ in OHS vs OSPS and $P=0.001$ in OHS vs ORPS, but $P=0.962$ in OSPS vs ORPS) (Figure 3B). However, all these findings were not found in the middle-aged comparison (MHS, MSPS, and MRPS, $P=0.211$ for 5-mc and $P=0.363$ in 5-hmc) or the younger-aged comparison (YHS, YSPS, and YRPS, $P=0.077$ for 5 -mc and $P=0.620$ in 5 -hmc) (data not shown).

In addition, in order to clarify the relationship of 5-mc and 5-hmc with age, we compared the levels among the three age groups in healthy controls, in the severe MDD group, and in the remitted MDD group. Trends of increasing in 5 -mc $(0.84 \% \pm 0.44 \%, 1.44 \% \pm 0.66 \%$, and $1.68 \% \pm 0.47 \%$ in YHS, MHS, and OHS, respectively, $P=0.052$ in YHS vs OHS) but no significant change in 5 -hmc $(1.29 \% \pm 0.84 \%$, $1.84 \% \pm 1.16 \%$, and $2.52 \% \pm 0.76 \%$ in YHS, MHS, and OHS,
A

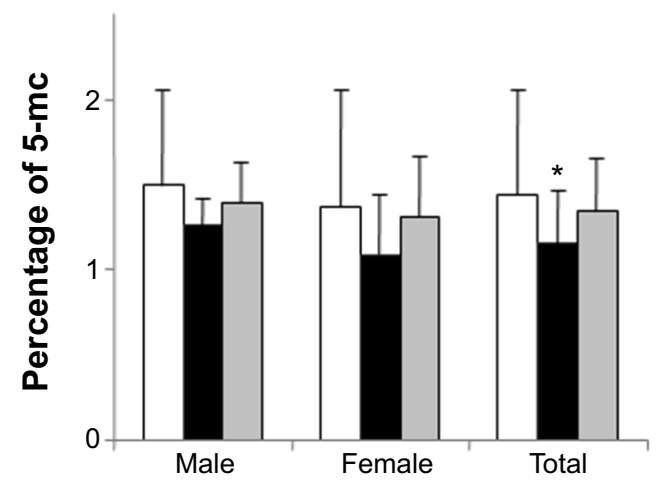

B

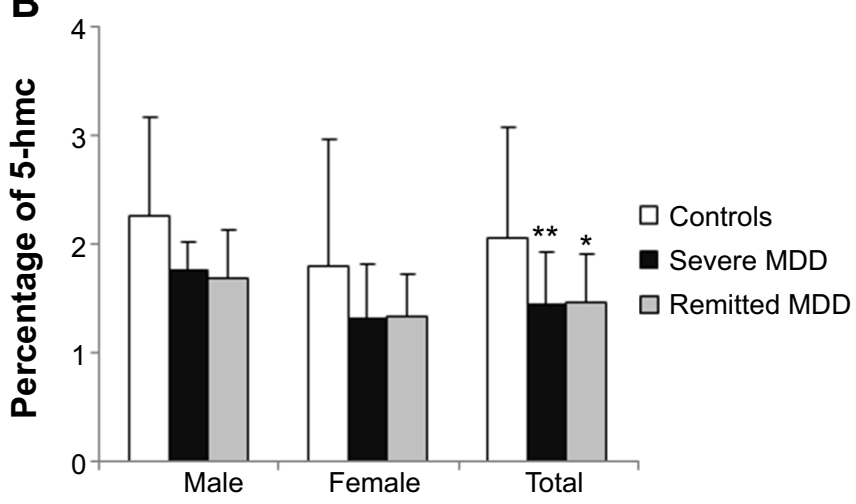

Figure 2 (A) 5-methylcytosine (5-mc) and (B) 5-hydroxymethylcytosine (5-hmc) levels in patients with severe major depressive disorder (MDD), remitted MDD, and healthy controls, divided by sex ( $n=30$ in males and $n=44$ in females).

Notes: The error bars are shown as standard deviations. The asterisks express significant difference from controls. $* P<0.05$, and $* * P<0.01$. 
A

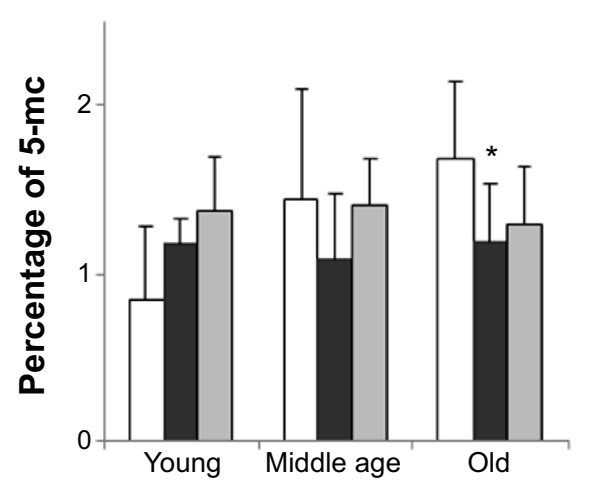

B

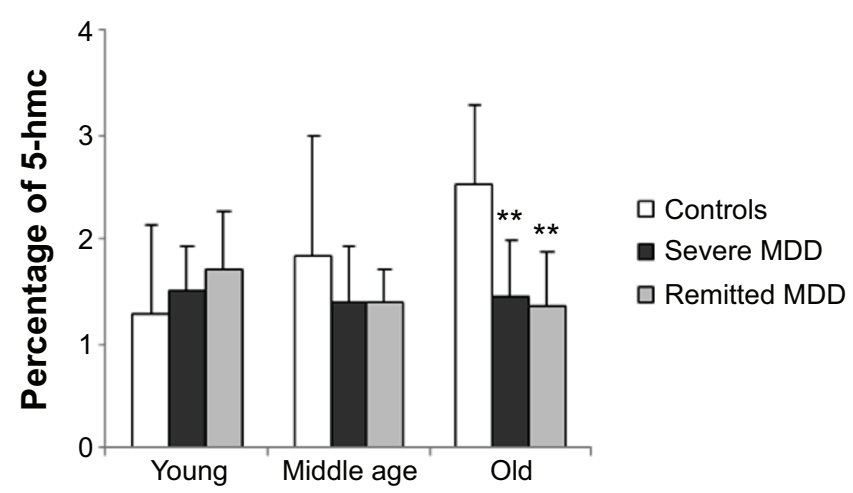

Figure 3 (A) 5-methylcytosine (5-mc) and (B) 5-hydroxymethylcytosine (5-hmc) levels in patients with severe major depressive disorder (MDD), remitted MDD, and healthy controls, in different age groups ( $n=14$ for younger group, $n=29$ for middle-age group, and $n=31$ for older age group).

Notes: Stratification of age groups are as follows: equal to or below 35 years of age, between 36 years and 50 years, and over 50 years. The error bars are shown as standard deviations. The asterisks denote significant difference from controls. $* P<0.05$ and $* * P<0.01$.

respectively, $P=0.111$ in YHS vs OHS) level with increased age were found in control subjects. But the increase did not appear in the severe MDD or remitted MDD patient subgroups comparison (for 5-mc: $P=0.791$ and 0.707, respectively; for 5-hmC: $P=0.948$ and 0.381 , respectively). These findings indicate that depressive disorder may impede natural increase during aging in 5-mc; but these findings could not be applied in 5-hmc levels.

\section{Correlation between 5-mc and 5-hmc levels and other clinical variables}

We found no correlation between 5-mc and 5-hmc levels and age $(P=0.133$ and 0.386 , respectively), illness onset $(P=0.243$ and 0.650 , respectively), illness duration $(P=0.131$ and 0.305 , respectively), years of education $(P=0.311$ and 0.088 , respectively), or BMI ( $P=0.254$ and 0.979 , respectively). However, there was a significant correlation between $5-\mathrm{mc}$ and 5-hmc levels $(r=0.804, P<0.001)$.

\section{Relationship between 5-mc and 5-hmc levels} and HAM-D scores

There was a significant inverse correlation between 5-mc level and HAM-D scores $(r=-0.360, P=0.011)$ (Figure 4A), but not in 5-hmc level $(r=-0.058, P=0.690)$ (Figure 4B).

\section{Relationship between 5-mc and 5-hmc levels} and psychotropic agents

We evaluated the effect of psychotropic agents on 5-mc and 5-hmc levels by comparing these levels between patients with or without such agents and examining the correlation
A

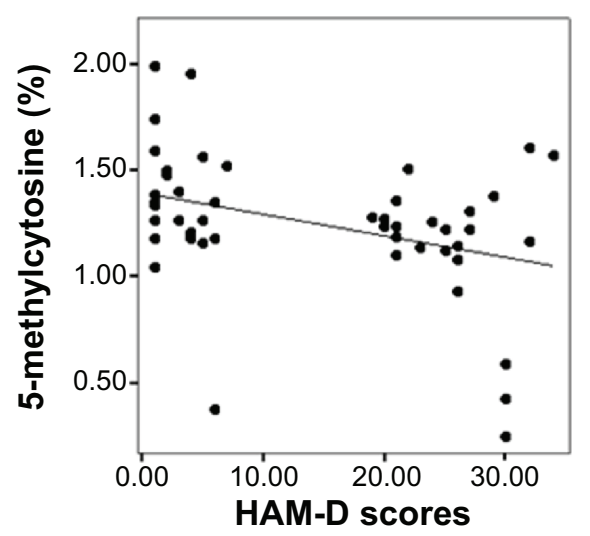

B

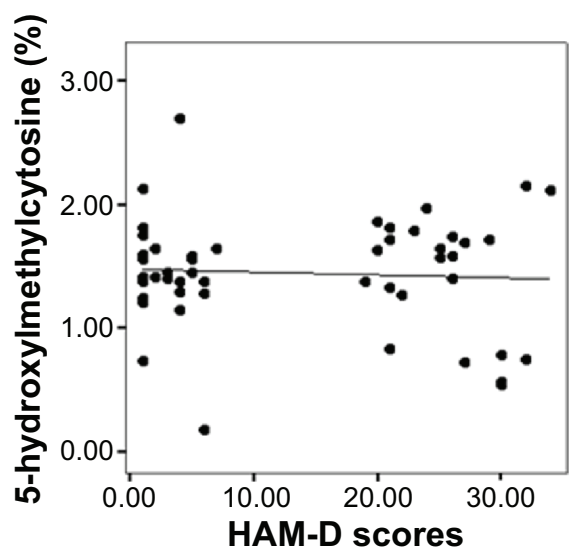

Figure 4 Scatterplots of the correlation between (A) 5-methylcytosine (5-mc) levels, and (B) 5-hydroxymethylcytosine (5-hmc) levels and the severity of depressive symptoms shown as HAM-D.

Notes: Pearson's correlation $r=-0.360, P=0.01$ I for 5 -mc and $r=-0.058, P=0.690$ for 5 -hmc.

Abbreviation: HAM-D, Hamilton Depression Rating Scale. 
between the levels and imipramine or chlorpromazine equivalent of psychotropic agents used by patients. We found a significantly higher 5-mc level in drug-free patients $(1.33 \% \pm 0.18 \%)$ than medicated patients $(1.04 \% \pm 0.35 \%)$ with severe $\operatorname{MDD}(P=0.031)$. However, there was no significant difference in 5 -hmc levels between drug-free and medicated patients $(P=0.897)$. There was no significant correlation between 5-mc and 5-hmc levels and imipramine equivalent ( $P=0.992$ and 0.725 , respectively) or chlorpromazine equivalent ( $P=0.972$ and 0.912 , respectively).

\section{Comparison of 5-mc and 5-hmc levels between first episode and recurrent MDD patients}

There were 17 first-episode MDD patients included in our study (seven in the severe MDD and ten in the remitted MDD group) and 32 patients with recurrent episodes (17 in severe and 15 in remitted MDD group). There was no significant difference in the 5-mc and 5-hmc levels between patients with first episode and with recurrent episodes, in either severe ( $d f=22, P=0.359$ for 5 -mc; $d f=22, P=0.753$ for 5 -hmc) or remitted ( $d f=23, P=0.688$ for $5-\mathrm{mc} ; d f=23, P=0.610$ for 5-hmc) MDD group.

\section{Comparison of 5-mc and 5-hmc levels between TRD and non-TRD patients}

There were six TRD patients in our study, all in the severe MDD group. We compared the 5-mc and 5-hmc levels between TRD and non-TRD patients with severe MDD. We found a decreasing trend in 5 -mc $(0.84 \% \pm 0.48 \%$ in TRD, $1.25 \% \pm 0.17 \%$ in non-TRD, $d f=22, P=0.092$ ) and $5-\mathrm{hmc}$ $(1.14 \% \pm 0.59 \%$ in TRD, $1.53 \% \pm 0.43 \%$ in non-TRD, $d f=22$, $P=0.090$ ) levels in TRD patients, compared to the non-TRD severe patient group.

\section{Comparison of 5-mc and 5-hmc levels in different sexes}

We examined the 5-mc and 5-hmc levels between the two sex subgroups in the different disease groups (male: $n=14,9$, and 7 in healthy controls, remitted MDD, and severe MDD groups, respectively; female: $\mathrm{n}=11,16$, and 17 in healthy controls, remitted MDD, and severe MDD groups, respectively) and found a significant difference in 5-hmc levels of the severe MDD groups only (male: $1.76 \% \pm 0.26 \%$ and female: $1.30 \% \pm 0.51 \%, d f=22, P=0.008)$ but not in the other groups (data not shown). To exclude the effect by disease state on 5-hmc, we examined the HAM-D scores between these two sex groups but found no significant difference $(P=0.471)$.

\section{Discussion}

In our study, we found significantly lower levels of 5-hmc and a trend of lower levels of 5-mc in peripheral leukocytes in patients with severe MDD than in healthy controls. However, no difference was found between patients with severe MDD and remitted patients. In addition, we also found a significant negative correlation between 5-mc level and the severity of depression. To our knowledge, this is the first report directly examining global methylation levels in different status of depression. Fuchikami et al had examined the DNA methylation profiles of the brain-derived neurotrophic factors $(B D N F)$ gene in peripheral blood of patients with MDD. They found that the methylation profiles of $\mathrm{CpG}$ I of the $B D N F$ gene might be a candidate diagnostic biomarker for MDD. ${ }^{8}$ In our current study, we emphasized the difference of global methylation levels in patients with different disease status of MDD. However, there were still some confounding factors in our study, which might influence the epigenetic profiles. For instance, the chlorpromazine equivalent was significantly higher in the severe MDD patient group than that in the remitted MDD patient group. Haloperidol, a typical antipsychotic drug, had been shown to induce epigenetic changes on peripheral leukocytes in a sex-dependent manner, namely, slightly decreased methyl cytosine in male rats and increased methyl cytosine in female rats. ${ }^{43}$ In addition, the education level was significant higher in the healthy control group than in the two patient groups. This may have resulted from the fact that our healthy controls were recruited from the self-paid general health examination center and may have higher socioeconomic status. In spite of those confounding factors, our study still suggests that 5-mc and 5-hmc might be the disease state biomarker of MDD.

Similar findings have been discussed in other psychiatric diseases. Shimabukuro et al had tested the methylation profiles of peripheral leukocyte DNA in patients with schizophrenia and found that there was a sex-dependent difference, with higher levels in methylated deoxycytidine content in males than in females. Also, there was a tendency toward lower methylated deoxycytidine content in patients than in healthy control subjects. ${ }^{42}$ However, the conclusion of hypomethylation in peripheral leukocytes of patients with schizophrenia is inconclusive. Bromberg et al found no difference in peripheral leukocyte methylation profile between patients with schizophrenia and healthy controls. ${ }^{44}$ Recently, one methylome-wide association study found some candidate sites of methylation in blood samples in patients with schizophrenia. ${ }^{45}$ Similar abnormal methylation profiles were also found by Kinoshita et al. ${ }^{46}$ Indeed, our results partially 
agreed with the sex-dependent difference of methylation profile found in the study by Shimabukuro et $\mathrm{al}^{42}$; however, there were significantly higher 5-hmc levels in men than in women, but not in 5-mc levels. This discrepancy might have been due to the different tools of detecting the methylation profiles and the different disease entities. In another study, Bromberg et al found that there was no difference in the methylation profiles of peripheral leukocytes between euthymic bipolar patients and healthy controls. ${ }^{47}$ In addition, studies have shown changes in global methylation levels by smoking ${ }^{48}$ and childhood maltreatment. ${ }^{4,49}$ These controversial results have suggested the importance in taking demographic and clinical variables into account when evaluating the feasibility of using epigenetic profiles in peripheral blood as biomarkers in psychiatric disorders.

In addition, we found a gradual increase in 5-mc levels during the aging process only in healthy controls, but not in severe or remitted MDD patients. In parallel, the significant difference of 5-mc levels between the healthy controls and MDD patients in our study existed only in the older subjects but not in the middle or younger age group. As reported by Gryzinska et al ${ }^{50}$ global methylation was found to increase with aging in a chicken model. Similarly, in the reports by Drinkwater et al and Golbus et $\mathrm{al}^{40,41}$ reduced DNA methylation level was detected in aging human leukocytes or T cells in an in vitro model. Increased methylation in the white matter of the brain of aged rhesus monkeys was also reported. ${ }^{51}$ Meanwhile, there is more and more evidence indicating increased methylation during aging, which might be, at least partially, caused by oxidative stress. ${ }^{52,53}$ These findings indicate that loss of genomic methylation may be involved in aging process. In parallel, Ben-Avraham et al reported that there was an increase in the total number of altered methylation sites with increasing age. ${ }^{54}$ Together with our findings, it might suggest that the MDD disease has the effect of diminishing or even erasing the trend of increasing methylation levels in the normal aging process. The diminishing or erasing effect might be stronger in patients with more severe depression, as evidenced by an inverse correlation between the HAM-D score and the leukocyte 5-mc level. However, more specifically designed studies are required to explore the relationship between DNA methylation and the normal aging process, and to confirm whether depression itself or psychotropic agents diminish the decrease in DNA methylation. On the contrary, there were no significantly different changes of the 5-hmc with aging in our study. Also, in another report using an animal model by Chen et al the 5-hmc in the mouse hippocampus significantly increased during aging. ${ }^{27}$
However, this result remains controversial. Decreased 5-hmc in the brain of mice during aging was found by Dzitoyeva et al. ${ }^{26}$ Therefore, we still need more evidence to clarify the trend of changes of 5-hmc during aging.

Moreover, we found significantly lower 5-hmc levels in patients with MDD than in healthy controls in our study. The 5-hmc is mainly found in the gene bodies of highly transcribed genes, ${ }^{55}$ or as playing a decisive role in the self-renewal process of embryonic stem cells. ${ }^{56}$ In clinical aspects, the 5-hmc was also found to be decreased in some cancers and had progressively lower levels along with the degree of malignancy. ${ }^{20,25}$ However, because of the lack of direct evidence showing any correlation of methylation levels between the peripheral environment and the central nervous system (CNS), we could not infer whether the lower levels of 5-hmc in the peripheral leukocytes of patients with depression could reflect the level in the CNS.

Some limitations need to be considered when interpreting our results. First, it is a cross-sectional study with a small sample size, and therefore, it is difficult to infer whether the methylation level will change during antidepressant treatment. Second, we did not take into account the risk factors of depression in our study, such as childhood maltreatment, recent life stressors, environmental factors, interactive effects of psychotropic drugs, and even nutritional status. Indeed, some of those factors have been shown to regulate methylation profiles. ${ }^{4,43,57,58}$ As mentioned above, there is a significant difference in chlorpromazine equivalents between the severe and remitted MDD patient subgroups. Indeed, this might have some effect on the methylation profile, not only by the directed effect on methylating process but also by the increasing lifetime of drug exposure due to the longer illness duration in severe MDD patients. However, because this is a cross-sectional study, we could not make sure the long-term drug compliance and the trends of effect on methylation by psychotropic drug use. Third, the patients of the severe MDD group recruited in our study had lower HAM-D scores than in previous studies. Actually, in studies conducted in the West, moderate MDD and severe MDD are generally defined as those with HAM-D scores of "between 18 and 25" and "not exceeding 25", separately. ${ }^{59,60}$ In addition, in our study, we could find out the impact of the disease severity on the 5-mc levels only as an inverse correlation. Hence, the relatively lower disease severity of MDD in our study might illustrate some effect on our results. Fourth, there is insufficient evidence showing any correlation between the peripheral and CNS methylation levels. It is difficult to determine whether any change in the methylation profile in peripheral leukocytes 
reflects that in CNS neurons. Further studies are needed to examine whether peripheral global methylation levels can be an age-associated biomarker of depression.

\section{Conclusion}

In summary, although we found a decrease in global DNA methylation in older patients with depression compared to healthy controls, this can possibly be explained by the fact that the normal increase in 5-mc and 5-hmc levels during aging is diminished or erased in depression. Also, the 5-mc level has an inverse correlation with depression severity in patients with MDD. It will be important to examine the role of the methylation level as a disease marker of depression and whether effective antidepressant treatment changes the methylation profiles.

\section{Acknowledgments}

We thank the patients, control subjects, and their families for participating in this study. This work was supported by the National Science Council, Taiwan (97-2321-B-182A-003-) and Chang Gung Memorial Hospital (CMRPG860271). These funding agencies had no role in the study design, collection of samples, analysis and interpretation of data, report writing, or decision to submit the article for publication.

\section{Author contributions}

PT Tseng assisted in the study design and data analyses, and wrote the initial draft. PY Lin initiated the study concept and design, interpreted statistical data, revised, and finalized the draft. CF Hung and Y Lee interviewed and assessed participants, helped with data collection, and revised the manuscript critically. FW Lung, CS Chen, and MY Chong advised in the study design, data interpretation, and critical revision. All authors read and approved the final manuscript, and agreed to be accountable for all aspects in this study.

\section{Disclosure}

The authors report no conflicts of interest in this work.

\section{References}

1. Sullivan PF, Neale MC, Kendler KS. Genetic epidemiology of major depression: review and meta-analysis. Am J Psychiatry. 2000;157(10):1552-1562.

2. Heim C, Binder EB. Current research trends in early life stress and depression: review of human studies on sensitive periods, geneenvironment interactions, and epigenetics. Exp Neurol. 2012;233(1): 102-111.

3. Weaver IC, Cervoni N, Champagne FA, et al. Epigenetic programming by maternal behavior. Nat Neurosci. 2004;7(8):847-854.

4. Perroud N, Paoloni-Giacobino A, Prada P, et al. Increased methylation of glucocorticoid receptor gene (NR3C1) in adults with a history of childhood maltreatment: a link with the severity and type of trauma. Transl Psychiatry. 2011;1:e59.
5. Jiao J, Opal MD, Dulawa SC. Gestational environment programs adult depression-like behavior through methylation of the calcitonin generelated peptide gene. Mol Psychiatry. 2013;18(12):1273-1280.

6. Uddin M, Koenen KC, Aiello AE, Wildman DE, de los Santos R, Galea S. Epigenetic and inflammatory marker profiles associated with depression in a community-based epidemiologic sample. Psychol Med. 2011;41(5):997-1007.

7. Rotter A, Asemann R, Decker A, Kornhuber J, Biermann T. Orexin expression and promoter-methylation in peripheral blood of patients suffering from major depressive disorder. J Affect Disord. 2011; 131(1-3):186-192.

8. Fuchikami M, Morinobu S, Segawa M, et al. DNA methylation profiles of the brain-derived neurotrophic factor (BDNF) gene as a potent diagnostic biomarker in major depression. PLoS One. 2011;6(8): e23881.

9. Zill P, Baghai TC, Schüle C, et al. DNA methylation analysis of the angiotensin converting enzyme (ACE) gene in major depression. PLoS One. 2012;7(7):e40479.

10. Sun HS, Kennedy PJ, Nestler EJ. Epigenetics of the depressed brain: role of histone acetylation and methylation. Neuropsychopharmacology. 2013;38(1):124-137.

11. Booij L, Wang D, Levesque ML, Tremblay RE, Szyf M. Looking beyond the DNA sequence: the relevance of DNA methylation processes for the stress-diathesis model of depression. Philos Trans $R$ Soc Lond B Biol Sci. 2013;368(1615):20120251.

12. Bestor TH. The DNA methyltransferases of mammals. Hum Mol Genet. 2000;9(16):2395-2402.

13. Wu H, Coskun V, Tao J, et al. Dnmt3a-dependent nonpromoter DNA methylation facilitates transcription of neurogenic genes. Science. 2010;329(5990):444-448.

14. Tahiliani M, Koh KP, Shen Y, et al. Conversion of 5-methylcytosine to 5-hydroxymethylcytosine in mammalian DNA by MLL partner TET1. Science. 2009;324(5929):930-935.

15. Valinluck V, Tsai HH, Rogstad DK, Burdzy A, Bird A, Sowers LC. Oxidative damage to methyl-CpG sequences inhibits the binding of the methyl-CpG binding domain (MBD) of methyl-CpG binding protein 2 (MeCP2). Nucleic Acids Res. 2004;32(14):4100-4108.

16. Fuks F, Hurd PJ, Wolf D, Nan X, Bird AP, Kouzarides T. The methylCpG-binding protein MeCP2 links DNA methylation to histone methylation. J Biol Chem. 2003;278(6):4035-4040.

17. Free A, Wakefield RI, Smith BO, Dryden DT, Barlow PN, Bird AP. DNA recognition by the methyl-CpG binding domain of $\mathrm{MeCP} 2 . J$ Biol Chem. 2001;276(5):3353-3360.

18. Kimura H, Shiota K. Methyl-CpG-binding protein, $\mathrm{MeCP} 2$, is a target molecule for maintenance DNA methyltransferase, Dnmt1.J Biol Chem. 2003;278(7):4806-4812.

19. Valinluck V, Sowers LC. Endogenous cytosine damage products alter the site selectivity of human DNA maintenance methyltransferase DNMT1. Cancer Res. 2007;67(3):946-950.

20. Jin SG, Wu X, Li AX, Pfeifer GP. Genomic mapping of 5-hydroxymethylcytosine in the human brain. Nucleic Acids Res. 2011;39(12): 5015-5024.

21. Rudenko A, Dawlaty MM, Seo J, et al. Tetl is critical for neuronal activityregulated gene expression and memory extinction. Neuron. 2013;79(6): 1109-1122.

22. Frycz BA, Murawa D, Borejsza-Wysocki M, et al. Decreased expression of ten-eleven translocation 1 protein is associated with some clinicopathological features in gastric cancer. Biomed Pharmacother. 2014;68(2):209-212.

23. Jin SG, Jiang Y, Qiu R, et al. 5-hydroxymethylcytosine is strongly depleted in human cancers but its levels do not correlate with IDH1 mutations. Cancer Res. 2011;71(24):7360-7365.

24. Kudo Y, Tateishi K, Yamamoto K, et al. Loss of 5-hydroxymethylcytosine is accompanied with malignant cellular transformation. Cancer Sci. 2012; 103(4):670-676.

25. Lian CG, Xu Y, Ceol C, et al. Loss of 5-hydroxymethylcytosine is an epigenetic hallmark of melanoma. Cell. 2012;150(6):1135-1146. 
26. Dzitoyeva S, Chen H, Manev H. Effect of aging on 5-hydroxymethylcytosine in brain mitochondria. Neurobiol Aging. 2012;33(12): 2881-2891.

27. Chen H, Dzitoyeva S, Manev H. Effect of aging on 5-hydroxymethylcytosine in the mouse hippocampus. Restor Neurol Neurosci. 2012;30(3):237-245.

28. Nagy C, Suderman M, Yang J, et al. Astrocytic abnormalities and global DNA methylation patterns in depression and suicide. Mol Psychiatry. Epub 2014 Mar 25.

29. Davies MN, Krause L, Bell JT, et al; UK Brain Expression Consortium. Hypermethylation in the ZBTB20 gene is associated with major depressive disorder. Genome Biol. 2014;15(4):R56.

30. Tseng PT, Lee Y, Lin PY. Age-associated decrease in serum glial cell line-derived neurotrophic factor levels in patients with major depressive disorder. Prog Neuropsychopharmacol Biol Psychiatry. 2013;40:334-339.

31. Dunner DL, Lipschitz A, Pitts CD, Davies JT. Efficacy and tolerability of controlled-release paroxetine in the treatment of severe depression: post hoc analysis of pooled data from a subset of subjects in four double-blind clinical trials. Clin Ther. 2005;27(12):1901-1911.

32. Arnold LM, Meyers AL, Sunderajan P, et al. The effect of pain on outcomes in a trial of duloxetine treatment of major depressive disorder. Ann Clin Psychiatry. 2008;20(4):187-193.

33. Lee MS, Ahn YM, Chung S, Walton R, Raskin J, Kim MS. The effect of initial duloxetine dosing strategy on nausea in Korean patients with major depressive disorder. Psychiatry Investig. 2012;9(4):391-399.

34. Wang Y, Fang YR, Chen XS, et al. A follow-up study on features of sensory gating P50 in treatment-resistant depression patients. Chin Med $J$ (Engl). 2009;122(24):2956-2960.

35. Cheng TA, Wu JT, Chong MY, Williams P. Internal consistency and factor structure of the Chinese Health Questionnaire. Acta Psychiatr Scand. 1990;82(4):304-308.

36. Chong MY, Wilkinson G. Validation of 30- and 12-item versions of the Chinese Health Questionnaire (CHQ) in patients admitted for general health screening. Psychol Med. 1989;19(2):495-505.

37. Souery D, Amsterdam J, de Montigny C, et al. Treatment resistant depression: methodological overview and operational criteria. Eur Neuropsychopharmacol. 1999;9(1-2):83-91.

38. Bollini P, Pampallona S, Tibaldi G, Kupelnick B, Munizza C. Effectiveness of antidepressants. Meta-analysis of dose-effect relationships in randomised clinical trials. $B r J$ Psychiatry. 1999; 174:297-303

39. Woods SW. Chlorpromazine equivalent doses for the newer atypical antipsychotics. J Clin Psychiatry. 2003;64(6):663-667.

40. Drinkwater RD, Blake TJ, Morley AA, Turner DR. Human lymphocytes aged in vivo have reduced levels of methylation in transcriptionally active and inactive DNA. Mutat Res. 1989;219(1):29-37.

41. Golbus J, Palella TD, Richardson BC. Quantitative changes in T cell DNA methylation occur during differentiation and ageing. Eur $J$ Immunol. 1990;20(8):1869-1872.

42. Shimabukuro M, Sasaki T, Imamura A, et al. Global hypomethylation of peripheral leukocyte DNA in male patients with schizophrenia: a potential link between epigenetics and schizophrenia. $J$ Psychiatr Res. 2007;41(12):1042-1046.

43. Shimabukuro M, Jinno Y, Fuke C, Okazaki Y. Haloperidol treatment induces tissue- and sex-specific changes in DNA methylation: a control study using rats. Behav Brain Funct. 2006;2:37.

Neuropsychiatric Disease and Treatment

\section{Publish your work in this journal}

Neuropsychiatric Disease and Treatment is an international, peerreviewed journal of clinical therapeutics and pharmacology focusing on concise rapid reporting of clinical or pre-clinical studies on a range of neuropsychiatric and neurological disorders. This journal is indexed on PubMed Central, the 'PsycINFO' database and CAS,
44. Bromberg A, Levine J, Nemetz B, Belmaker RH, Agam G. No association between global leukocyte DNA methylation and homocysteine levels in schizophrenia patients. Schizophr Res. 2008;101(1-3):50-57.

45. Aberg KA, McClay JL, Nerella S, et al. Methylome-wide association study of schizophrenia: identifying blood biomarker signatures of environmental insults. JAMA Psychiatry. 2014;71(3):255-264.

46. Kinoshita M, Numata S, Tajima A, et al. Aberrant DNA methylation of blood in schizophrenia by adjusting for estimated cellular proportions. Neuromolecular Med. Epub 2014 Jul 23.

47. Bromberg A, Bersudsky Y, Levine J, Agam G. Global leukocyte DNA methylation is not altered in euthymic bipolar patients. J Affect Disord. 2009;118(1-3):234-239.

48. Hillemacher T, Frieling H, Moskau S, et al. Global DNA methylation is influenced by smoking behaviour. Eur Neuropsychopharmacol. 2008;18(4):295-298.

49. Mehta D, Klengel T, Conneely KN, et al. Childhood maltreatment is associated with distinct genomic and epigenetic profiles in posttraumatic stress disorder. Proc Natl Acad Sci U S A. 2013;110(20):8302-8307.

50. Gryzinska M, Blaszczak E, Strachecka A, Jezewska-Witkowska G. Analysis of age-related global DNA methylation in chicken. Biochem Genet. 2013;51(7-8):554-563.

51. King GD, Rosene DL, Abraham CR. Promoter methylation and agerelated downregulation of Klotho in rhesus monkey. Age (Dordr). 2012;34(6):1405-1419.

52. Sundar IK, Yao H, Rahman I. Oxidative stress and chromatin remodeling in chronic obstructive pulmonary disease and smoking-related diseases. Antioxid Redox Signal. 2013;18(15):1956-1971.

53. Sanchez-Roman I, Gómez A, Pérez I, et al. Effects of aging and methionine restriction applied at old age on ROS generation and oxidative damage in rat liver mitochondria. Biogerontology. 2012;13(4): 399-411.

54. Ben-Avraham D, Muzumdar RH, Atzmon G. Epigenetic genomewide association methylation in aging and longevity. Epigenomics. 2012;4(5):503-509.

55. Szulwach KE, Li X, Li Y, et al. 5-hmC-mediated epigenetic dynamics during postnatal neurodevelopment and aging. Nat Neurosci. 2011; 14(12):1607-1616.

56. Ito S, D'Alessio AC, Taranova OV, Hong K, Sowers LC, Zhang Y. Role of Tet proteins in $5 \mathrm{mC}$ to $5 \mathrm{hmC}$ conversion, ES-cell self-renewal and inner cell mass specification. Nature. 2010;466(7310):1129-1133.

57. Melas PA, Rogdaki M, Lennartsson A, et al. Antidepressant treatment is associated with epigenetic alterations in the promoter of P11 in a genetic model of depression. Int J Neuropsychopharmacol. 2012;15(5): 669-679.

58. Uher R. Genes, environment, and individual differences in responding to treatment for depression. Harv Rev Psychiatry. 2011;19(3): 109-124.

59. Jevtovic S, Karlovic D, Mihaljevic-Peles A, Seric V, Vrkic N, Jaksic N. Serum Brain-derived neurotrophirc factor (Bdnf): the severity and symptomatic dimensions of depression. Psychiatr Danub. 2011;23(4): 363-369.

60. Muller MJ, Himmerich H, Kienzle B, Szegedi A. Differentiating moderate and severe depression using the Montgomery-Asberg depression rating scale (MADRS). J Affect Disord. 2003;77(3):255-260.

and is the official journal of The International Neuropsychiatric Association (INA). The manuscript management system is completely online and includes a very quick and fair peer-review system, which is all easy to use. Visit http://www.dovepress.com/testimonials.php to read real quotes from published authors. 